\title{
Molecular Characterization of a Rare Case of Bilateral Vitreoretinal T Cell Lymphoma through Vitreous Liquid Biopsy
}

\author{
Andi K. Cani ${ }^{1,2, \dagger}{ }^{\dagger}$, Marcus A. Toral ${ }^{3,4,5, \dagger}$, Daniel A. Balikov ${ }^{6,+}{ }^{-}$, Bryan L. Betz ${ }^{7}$, Kevin Hu ${ }^{7,8}$, Chia-Jen Liu ${ }^{7,9}$, \\ Matthew V. Prifti 10,11, Arul M. Chinnaiyan 2,7,9, Scott A. Tomlins 7,9, Vinit B. Mahajan 5,12,* \\ and Rajesh C. Rao ${ }^{2,6,7,8,10,13, *(\mathbb{D})}$
}

check for updates

Citation: Cani, A.K.; Toral, M.A.; Balikov, D.A.; Betz, B.L.; Hu, K.; Liu, C.-J.; Prifti, M.V.; Chinnaiyan, A.M.; Tomlins, S.A.; Mahajan, V.B.; et al.

Molecular Characterization of a Rare Case of Bilateral Vitreoretinal T Cell Lymphoma through Vitreous Liquid Biopsy. Int. J. Mol. Sci. 2021, 22, 6099. https://doi.org/10.3390/ijms22116099

Academic Editor: Evgeny Imyanitov

Received: 26 April 2021

Accepted: 30 May 2021

Published: 5 June 2021

Publisher's Note: MDPI stays neutral with regard to jurisdictional claims in published maps and institutional affiliations.

Copyright: (c) 2021 by the authors. Licensee MDPI, Basel, Switzerland. This article is an open access article distributed under the terms and conditions of the Creative Commons Attribution (CC BY) license (https:// creativecommons.org/licenses/by/ $4.0 /)$.
1 Department of Internal Medicine, University of Michigan, Ann Arbor, MI 48105, USA; acani@med.umich.edu Rogel Cancer Center, University of Michigan, Ann Arbor, MI 48109, USA; arul@med.umich.edu Medical Scientist Training Program, University of Iowa, Iowa City, IA 52242, USA; marcustoral7@gmail.com Graduate Program in Molecular Medicine, University of Iowa, Iowa City, IA 52242, USA

Molecular Surgery Laboratory, Byers Eye Institute, Stanford University, Palo Alto, CA 94303, USA

6 W.K. Kellogg Eye Center, Department of Ophthalmology and Visual Science, University of Michigan, Ann Arbor, MI 48105, USA; dbalikov@med.umich.edu

7 Department of Pathology, University of Michigan, Ann Arbor, MI 48109, USA; bbetz@med.umich.edu (B.L.B.); kevhu@med.umich.edu (K.H.); jiuchiaj@med.umich.edu (C.-J.L.); scott@strataoncology.com (S.A.T.)

8 Department of Computational Medicine and Bioinformatics, University of Michigan, Ann Arbor, MI 48109, USA

9 Michigan Center for Translational Pathology, University of Michigan, Ann Arbor, MI 48109, USA

10 A. Alfred Taubman Medical Research Institute, University of Michigan, Ann Arbor, MI 48105, USA; matthew.prifti@wayne.edu

11 Department of Biological Sciences, Wayne State University, Detroit, MI 48202, USA

12 Palo Alto Veterans Health Care System, Palo Alto, CA 94304, USA

13 Division of Ophthalmology, Surgical Service, Veterans Administration Ann Arbor Healthcare System, Ann Arbor, MI 48105, USA

* Correspondence: vinit.mahajan@stanford.edu (V.B.M.); rajeshr@med.umich.edu (R.C.R.); Tel.: +1-650-723-6995 (V.B.M.); +1-734-647-1226 (R.C.R.);

Fax: +1-650-498-1528 (V.B.M.); +1-734-232-8030 (R.C.R.)

+ Co-first authors.

Abstract: Vitreoretinal lymphoma (VRL) is an uncommon eye malignancy, and VRLs of T cell origin are rare. They are difficult to treat, and their molecular underpinnings, including actionable genomic alterations, remain to be elucidated. At present, vitreous fluid liquid biopsies represent a valuable VRL sample for molecular analysis to study VRLs. In this study, we report the molecular diagnostic workup of a rare case of bilateral T cell VRL and characterize its genomic landscape, including identification of potentially targetable alterations. Using next-generation sequencing of vitreous-derived DNA with a pan-cancer 126-gene panel, we found a copy number gain of BRAF and copy number loss of tumor suppressor DNMT3A. To the best of our knowledge, this represents the first exploration of the T cell VRL cancer genome and supports vitreous liquid biopsy as a suitable approach for precision oncology treatments.

Keywords: precision oncology; next-generation sequencing; liquid biopsy; vitreoretinal lymphoma; T cell lymphoma

\section{Introduction}

Vitreoretinal lymphoma (VRL), the most common ocular lymphoma, is often associated with primary CNS lymphoma (PCNSL, up to $90 \%$ ) from where it is thought to spread to the eye [1,2]. VRL is rare ( 380 US cases/year) and presents most commonly as diffuse large B cell lymphoma (DLBCL) [1]. VRL can cause vision loss and has a poor prognosis. Radio- and chemotherapy are used empirically and have short-lived responses (progression-free survival (PFS) 1-year, overall survival (OS) 3 years). Genomic driver 
alterations have been described in a limited number of patients by us and others, but precision oncology approaches that use molecularly informed targeted treatments remain in their infancy $[3,4]$.

Diagnostically, vitreous fluid-sampled VRL cells are analyzed by cytology and/or flow cytometry for morphological features and cell surface markers. However, cell scarcity, limited fluid amount, and the gelatinous consistency of the fluid contribute to inaccurate results $[5,6]$. High-sensitivity assays, such as the detection of the lymphocyte cell receptor $\mathrm{V}(\mathrm{D}) \mathrm{J}$ DNA recombination characteristic of clonal lymphoid populations or the use of nextgeneration sequencing (NGS) to identify driver genomic alterations, are highly compatible with vitreous liquid biopsy [3] obtained on an outpatient basis or during vitrectomy. However, these molecular assays, especially genomic analysis, are not routinely performed.

While the majority of PCNSL/VRL cases are DLBCLs, an even rarer subset belongs to the $\mathrm{T}$ cell subtype. Herein, we report for the first time to our knowledge the molecular diagnostic and genomic analysis of vitreous liquid biopsy samples from a bilateral $\mathrm{T}$ cell VRL case in a 63-year-old male. Following a pioneering approach we previously developed for B cell VRL genomic analysis and precision medicine target nomination from vitreous liquid biopsy [3], herein we used a targeted NGS gene panel (Oncomine Cancer Panel [7], one of the assays used in the NCI-MATCH targeted therapy basket trial [8]) to analyze VRL DNA from this patient.

\section{Methods}

\subsection{Case Selection}

The study was conducted with approval from the IRBs of the University of Michigan (UM) and the University of Iowa (UI). We identified a case of T cell VRL in a 63-year-old male who underwent therapeutic vitrectomy. Vitreous samples were genomically analyzed at the UM Kellogg Eye Center and Pathology Department. Clinicopathological information was obtained from the clinical archive.

\subsection{DNA Analysis}

Approximately $3.5 \mathrm{~mL}$ of undiluted vitreous fluid from each eye, stored at $-80^{\circ} \mathrm{C}$, was centrifuged at $1000 \times g$ for $5 \mathrm{~min}$. Genomic DNA was extracted from the resulting pellets using the Qiagen AllPrep FFPE DNA/RNA kit (Qiagen, Hilden, Germany), with the following modifications for non-FFPE cells: (1) no de-paraffinization treatment; (2) first $56{ }^{\circ} \mathrm{C}$ incubation reduced to $2 \mathrm{~min}$; (3) incubation at $90{ }^{\circ} \mathrm{C}$ omitted). DNA samples were quantitated with the Qubit Fluorometer (ThermoFisher, Waltham, MA, USA). T cell receptor (TCR) rearrangement testing was performed with the BIOMED-2 PCR assays (Invivoscribe Technologies, Inc., San Diego, CA, USA) covering 90\% of TCRB and TCRG rearrangements.

\subsection{Next-Generation Sequencing}

NGS was performed as previously described [3,9]. Briefly, $20 \mathrm{ng}$ of DNA underwent library construction using a targeted custom panel (Oncomine Cancer Panel (OCP), ThermoFisher) targeting 126 cancer-related genes (3435 amplicons). Targets were selected based on large-scale pan-solid tumor and lymphoma genomic data prioritizing recurrent and/or targetable cancer mutations, short insertions/deletions, and copy number alterations (CNAs) [7]. Sequencing and analysis were performed as previously reported $[7,10]$. Briefly, barcoded libraries were constructed with the AmpliSeq method (Library Kit 2.0, ThermoFisher). Sequencing was performed on IonProton and Torrent Suite 5.0.2. Variant and CNA annotation, filtering, and prioritization were performed as reported using in-house pipelines.

\subsection{Next-Generation Sequencing Analysis}

Data analysis was performed using Torrent Suite 5.0.2, with alignment by TMAP using default parameters, and variant calling was performed using the Torrent Variant 
Caller plugin (version 4.0-r76860) using default low-stringency somatic variant settings. Called variants were filtered to remove synonymous or non-coding variants, those with flow-corrected read depths (FDP) $<20$, flow-corrected variant allele containing reads (FAO) $<6$, variant allele fractions $(\mathrm{FAO} / \mathrm{FDP})<0.10$, extreme skewing of forward/reverse flowcorrected reads calling the variant (FSAF/FSAR $<0.2$ or $>5$ ), or indels within homopolymer runs $>4$. Called variants were then filtered using a panel-specific, in-house blacklist. Variants with allele frequencies $>0.5 \%$ in the EXAC database and those reported in EXAC and with observed variant allele fractions between 0.40 and 0.60 or $>0.9$ were considered germline variants unless occurring at a known hot-spot. Variants located at the last mapped base (or outside) of amplicon target regions, variants with the majority of supporting reads harboring additional mismatches or indels (likely sequencing errors), those in repeat-rich regions (likely mapping artifacts), and variants occurring exclusively in one amplicon if overlapping amplicons cover the variant, were excluded. High-confidence somatic variants passing the above criteria were then visually confirmed in Integrative Genomics Viewer (Broad Institute, Cambridge, MA, USA, https:/ / www.broadinstitute.org/igv / (accessed on 15 October 2020)). We have previously confirmed that these filtering criteria identify prioritized high-confidence somatic variants that pass Sanger sequencing validation with $>95 \%$ accuracy [11].

Copy number analysis from total amplicon read counts provided by the Coverage Analysis Plug-in (v4.0-r77897) was performed essentially as described using a validated in-house approach. Log2 copy number ratio was calculated as the amplicon level ratio between read counts in the tumor sample and read counts in a composite of normal samples, normalized for sequencing depth and GC content. Gene-level estimates were calculated as coverage-weighted averages of amplicon-level $\log 2 \mathrm{CN}$ ratios. Genes with a $\log 2$ copy number ratio estimate of $<-1$ or $>0.6$ were considered to have a high level of loss or gain, respectively.

\section{Results}

A 63-year-old male with a past medical history of shingles and anterior uveitis in the left eye six months prior, presented with blurred vision and floaters in both eyes. Other baseline workup reported no relative afferent pupillary defect (diagnostic sign for optic nerve pathology) and normal intraocular pressure (diagnostic sign for pressureinduced vision changes). Most notably, the patient's vitreous humor contained free-floating cells in both eyes (where there should be none), and the left eye demonstrated whitish deposits in the subretinal space of the central and peripheral retina (Figure 1A-H). PCR of anterior aqueous ocular fluid was negative for herpes simplex virus, cytomegalovirus, or varicella zoster virus infection, ruling out an infectious herpes zoster related etiology. The patient underwent sequential diagnostic and therapeutic vitrectomy, at which time, $3.5 \mathrm{~mL}$ of undiluted vitreous was stored for future cytologic and genomic analysis. The patient reported improved symptoms and postoperative visual acuity of perfect vision in both eyes.

Cytology revealed mononuclear cells that were CD20- and CD10-negative and CD3positive, suggesting vitreous T cell presence (Figure 1I). Genomic DNA (66 ng (OS, oculus sinister, left eye) and $37 \mathrm{ng}(\mathrm{OD}$, oculus dexter, right eye) was extracted from the undiluted vitreous and underwent T cell receptor (TCR) PCR testing. This assay has the sensitivity to detect the $90 \%$ of most common V(D)J rearrangements in the TCRB and TCRG genes [12]. Nearly identical peaks of $186.02 \mathrm{bp}$ (OS) and $186.04 \mathrm{bp}$ (OD) were present at $\sim 5-10 \%$ frequency, indicating the presence of clonal $\mathrm{T}$ cell populations (Figure 2A). These peaks were present only in the TCRG assay. 


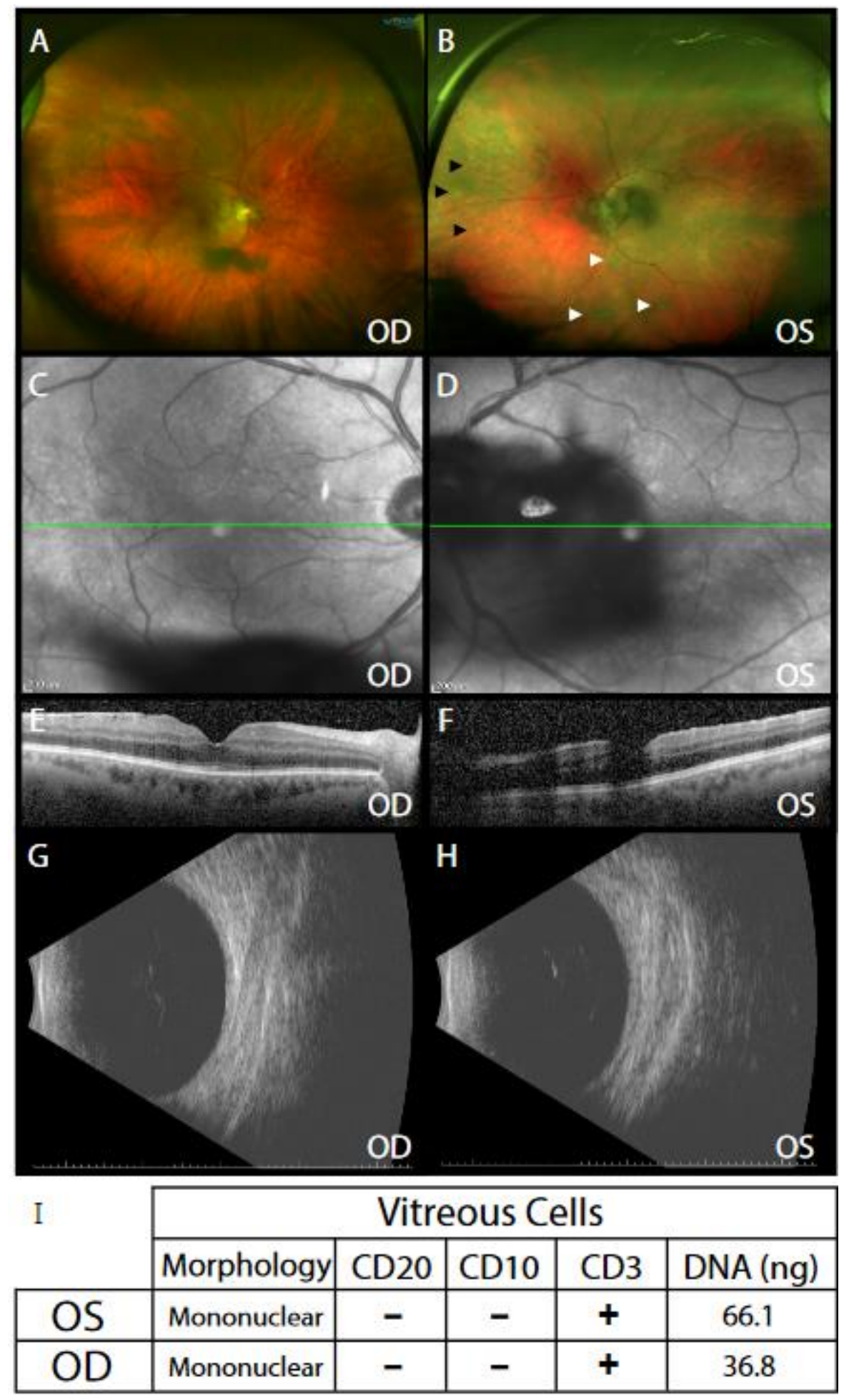

Figure 1. Clinical examination shows vitreous debris in both eyes. (A) Ultra-widefield retinal imaging of the right eye (OD) showed vitreous clumps over the posterior pole. (B) Ultra-widefield retinal imaging of the left eye (OS) showed vitreous cells (black arrowheads); inferior snowballs (white arrowheads); and dense vitreous clumps over the posterior pole. (C,D) Infrared en-face imaging shows level (green-line) at which optical coherence tomography (OCT) was used to image cross-section of macula (E,F). Cross-sectional OCT showed (C) mild epiretinal membrane OD and (D) a dense vitreous opacity over the macula OS. Posterior B-Scan ultrasonography revealed vitreous opacities (G) OD and (H) OS. (I) Cytological examination for morphology and cell-surface marker staining for CD20, CD10 and CD3 as well as vitreous DNA amounts are shown for each vitreous sample. 
A

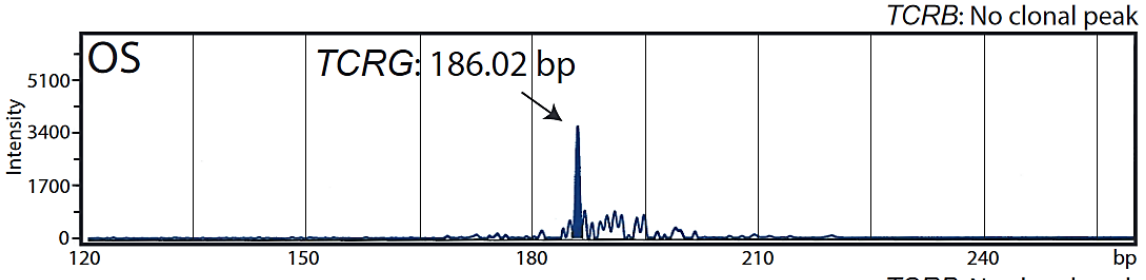

TCRB: No clonal peak

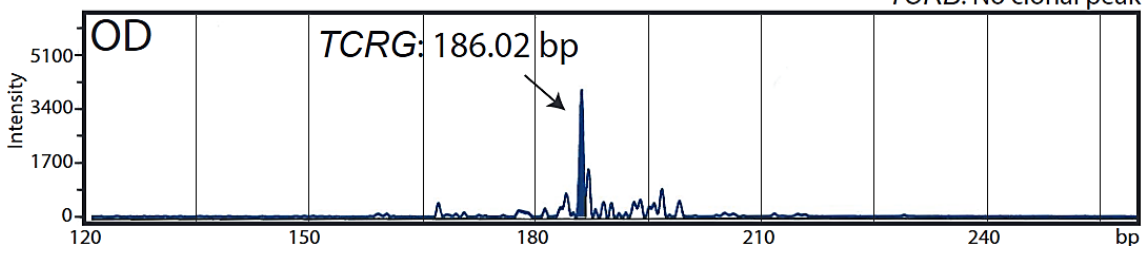

B
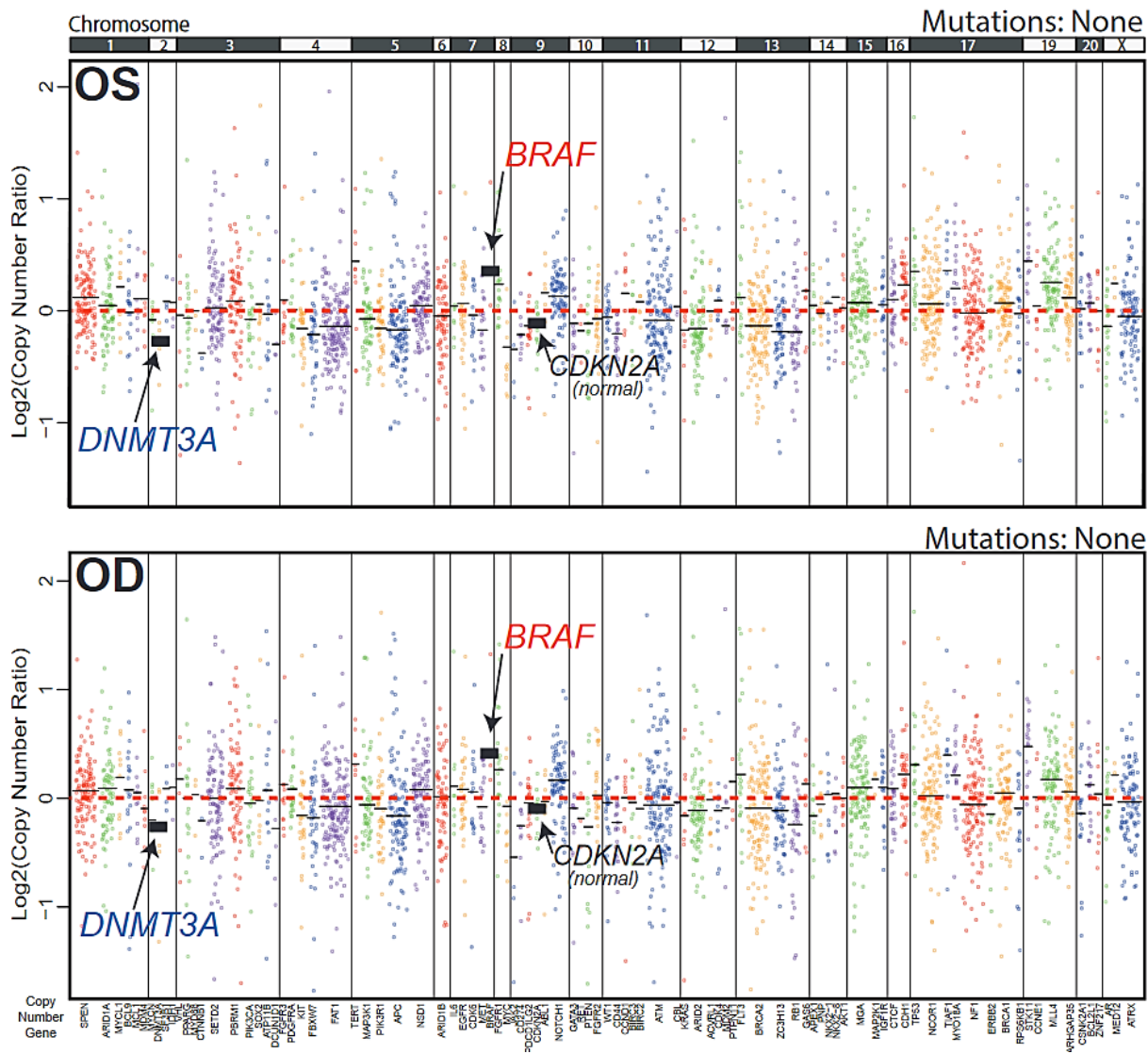

Figure 2. DNA analysis of vitreous liquid biopsy samples. (A) DNA obtained from vitreous fluid from each eye underwent clonal T cell receptor (TCR) rearrangement PCR testing covering $~ 90 \%$ of the most common rearrangements in the TCRB and TCRG genes. Assays were run in duplicate with negative controls (one replicate shown per sample). Clonal rearrangements were defined as peaks identical between replicates that were at least $2 \times$ higher than the third highest peak. Capillary electropherogram plots of band intensity over fragment length show a positive PCR result for TCRG rearrangement at $186 \mathrm{bp}$, identical in both eyes, at $10 \%$ frequency. No TCRB-positive bands were observed in either sample. (B) NGS using the OCP version 1 panel was performed on each eye sample. Copy number plots are shown as log2 copy number ratio (amplicon level ratio between read counts in the tumor sample and read counts in a composite of normal samples, normalized for sequencing depth and GC content). Dots represent individual amplicons, dots of the same color represent a gene, and black horizontal bars represent average gene-level estimates (coverageweighted). Altered/relevant genes are highlighted. No mutations were observed in either sample. 
In order to rule out a non-malignant clonal $\mathrm{T}$ cell proliferation, (e.g., infection), NGS using a panel of 126 cancer-related genes, modified from the Oncomine Cancer Panel (OCP) used in the NCI MATCH clinical trial, was performed. Both OS and OD samples showed a scarcity of mutations and short insertions or deletions in the genes assayed. This included a lack of MYD88 hotspot mutations commonly observed in vitreoretinal and systemic B cell lymphomas. However, copy number alterations (CNAs) were present in nearly identical patterns between the two eyes. These were characterized by a copy gain in the oncogene $B R A F$, an actionable alteration. Genes with copy number loss included the DNA methyltransferase DNMT3A, a tumor suppressor and epigenetic DNA-modifying enzyme. Neither sample showed a copy loss in the cell cycle inhibitor CDKN2A, a highly recurrent $B$ cell VRL alteration [3]. These CNAs were present at low levels, consistent with the low TCRG rearrangement peak predominance of $5-10 \%$ (Figure 2B). In order to rule out our observed CNAs being due to sequencing technical variability, we compared the nearly identical OD and OS copy number profiles to a sample obtained from another patient run in the same experiment and initially suspected for VRL but found not to harbor any evidence of cancer (Supplementary Figure S1, Sample \#7). OD/OS copy number profiles differed from Sample \#7, thus providing further support for OD and OS containing clonal malignant populations harboring $B R A F$ and DNMT3A CNAs. Taken together, these data show the feasibility of vitreous liquid biopsy as a source suitable not only for commonly performed morphological and surface marker detection, but also for obtaining precise molecular genomic information in VRL cases. This information supports unambiguous diagnostication and enables nomination of putative precision oncology biomarkers to expand treatment options.

\section{Discussion}

To our knowledge, this work represents the first exploration of the actionable cancer genome of a T cell VRL case. This patient showed a rare clonal T cell population, with no lymphoma seen outside of the eye, and was treated with eight cycles of bilateral intravitreal methotrexate. There was no recurrence and no CNS or systemic lymphoma seen during his disease course. Using NGS, we found a scarcity of mutations/indels but presence of CNAs in an oncogene and tumor suppressor.

Limitations include the "N-of-1" nature of this study which limits generalizability, as well as our limited targeted gene set which does not preclude alterations in genes that were not assayed here. An additional limitation is the low tumor content of both samples (as indicated by the TCRG clonal peak dominance of 5-10\% and modest CNA levels). Although the evidence suggests presence of clonal malignant $\mathrm{T}$ cell populations identical in each eye, a non-malignant cause cannot be definitively excluded based on the available data.

Our data suggest largely distinct genomic landscapes between B cell VRLs and this $T$ cell case in agreement with previous non-ocular, systemic lymphoma studies [3,13-16]. While CDKN2A deleterious alterations (highly recurrent in B cell lymphomas) have been shown to recur in systemic T cell lymphomas [16], our patient had a neutral copy number for this gene. TP53 alterations, also described in systemic T cell lymphomas [14,16], were absent in this patient. Taken together, our data suggest that T cell VRLs are amenable to genomic analysis for diagnosis and precision oncology from minute vitreous liquid biopsy samples.

Supplementary Materials: The following are available online at https:/ /www.mdpi.com/article/10 .3390/ijms22116099/s1, Figure S1: Copy number profile of a likely normal sample. 
Author Contributions: Conceptualization R.C.R., V.B.M. and S.A.T.; methodology A.K.C. and D.A.B.; formal analysis A.K.C., M.A.T., B.L.B., K.H. and C.-J.L.; investigation A.K.C., M.A.T., M.V.P. and B.L.B.; data curation A.K.C., M.A.T. and D.A.B.; writing-original draft preparation A.K.C. and M.A.T.; writing-review and editing A.K.C., M.A.T., D.A.B., B.L.B., A.M.C., S.A.T., V.B.M. and R.C.R.; visualization A.K.C. and M.A.T.; supervision A.M.C., S.A.T., V.B.M. and R.C.R.; funding acquisition A.M.C., S.A.T. and R.C.R. All authors have read and agreed to the published version of the manuscript.

Funding: AKC was supported by the NIH Training Program in Translational Research (T32GM113900) during parts of this study. During this study, RCR was supported by the National Eye Institute (K12EY022299, K08EY026654, R01EY030989), Research to Prevent Blindness (RPB), the Beatrice \& Reymont Paul Foundation, March Hoops to Beat Blindness, and Leonard G. Miller Endowed Professorship and Ophthalmic Research Fund at the Kellogg Eye Center. Additional support for this research was provided by Grossman, Elaine Sandman, Marek and Maria Spatz (endowed fund), Greenspon, Dunn, Avers, Boustikakis, Sweiden, and Terauchi research funds to RCR. The NCI Cancer Center Support Grant supported this work (NCI CCSG-P30CA046592). During the course of this study, RCR was the Leslie H. and Abigail S. Wexner Emerging Scholar and SAT is the A. Alfred Taubman Emerging Scholar, both of the A. Alfred Taubman Medical Research Institute, which supported, in part, this study. VBM is supported by NIH grants (R01EY031952, R01EY030151, R01EY031360, P30 EY026877]) and RPB.

Institutional Review Board Statement: The study was conducted according to the guidelines of the Declaration of Helsinki, and approved by the Institutional Review Boards of the University of Michigan (protocol code HUM00085419 and date of approval 17 September 2014) and the University of Iowa (protocol code 201007738 and date of approval 12 April 2016).

Informed Consent Statement: Informed consent was obtained from all subjects involved in the study.

Data Availability Statement: Data reported in this study are available upon request to author Rajesh C. Rao.

Acknowledgments: We would like to thank the patient, his family, and all medical personnel involved in his care.

Conflicts of Interest: S.A.T. has received travel support and had a separate sponsored research agreement with Compendia Bioscience/Life Technologies/ThermoFisher Scientific, which provided access to the sequencing panel used herein. S.A.T. is an employee and equity holder in Strata Oncology.

\section{References}

1. Chan, C.; Rubenstein, J.L.; Coupland, S.; Davis, J.L.; Harbour, J.W.; Johnston, P.B.; Cassoux, N.; Touitou, V.; Smith, J.R.; Batchelor, T.T.; et al. Primary Vitreoretinal Lymphoma: A Report from an International Primary Central Nervous System Lymphoma Collaborative Group Symposium. Oncologist 2011, 16, 1589-1599. [CrossRef] [PubMed]

2. Pulido, J.S.; Johnston, P.B.; Nowakowski, G.S.; Castellino, A.; Raja, H. Correction to: The diagnosis and treatment of primary vitreoretinal lymphoma: A review. Int. J. Retin. Vitr. 2018, 4, 22. [CrossRef] [PubMed]

3. Cani, A.K.; Hovelson, D.H.; Demirci, H.; Johnson, M.W.; Tomlins, S.A.; Rao, R.C. Next generation sequencing of vitreoretinal lymphomas from small-volume intraocular liquid biopsies: New routes to targeted therapies. Oncotarget 2016, 8, 7989-7998. [CrossRef] [PubMed]

4. Hirschhorn, J.W.; Snider, J.S.; Lindsey, K.G.; Schandl, C.A. Molecular profiling of vitreous fluid by massively parallel sequencing: A case series. J. Am. Soc. Cytopathol. 2020, 9, 254-257. [CrossRef] [PubMed]

5. Fend, F.; Ferreri, A.J.M.; Coupland, S. How we diagnose and treat vitreoretinal lymphoma. Br. J. Haematol. 2016, 173, 680-692. [CrossRef] [PubMed]

6. Davis, J.L. Intraocular lymphoma: A clinical perspective. Eye 2013, 27, 153-162. [CrossRef] [PubMed]

7. Hovelson, D.H.; McDaniel, A.S.; Cani, A.K.; Johnson, B.; Rhodes, K.; Williams, P.D.; Bandla, S.; Bien, G.; Choppa, P.; Hyland, F.; et al. Development and Validation of a Scalable Next-Generation Sequencing System for Assessing Relevant Somatic Variants in Solid Tumors. Neoplasia 2015, 17, 385-399. [CrossRef] [PubMed]

8. Conley, B.A.; Doroshow, J.H. Molecular Analysis for Therapy Choice: NCI MATCH. Semin. Oncol. 2014, 41, 297-299. [CrossRef] [PubMed]

9. Cani, A.K.; Soliman, M.; Hovelson, D.H.; Liu, C.-J.; McDaniel, A.S.; Haller, M.J.; Bratley, J.V.; Rahrig, S.; Li, Q.; Briceño, C.A.; et al. Comprehensive genomic profiling of orbital and ocular adnexal lymphomas identifies frequent alterations in MYD88 and chromatin modifiers: New routes to targeted therapies. Mod. Pathol. 2016, 29, 685-697. [CrossRef] [PubMed]

10. Grasso, C.S.; Cani, A.K.; Hovelson, D.H.; Quist, M.J.; Douville, N.J.; Yadati, V.; Amin, A.M.; Nelson, P.S.; Betz, B.L.; Liu, C.-J.; et al. Integrative molecular profiling of routine clinical prostate cancer specimens. Ann. Oncol. 2015, 26, 1110-1118. [CrossRef] 
11. Warrick, J.I.; Hovelson, D.H.; Amin, A.; Liu, C.-J.; Cani, A.K.; McDaniel, A.S.; Yadati, V.; Quist, M.J.; Weizer, A.Z.; Brenner, J.C.; et al. Tumor evolution and progression in multifocal and paired non-invasive/invasive urothelial carcinoma. Virchows Archiv 2015, 466, 297-311. [CrossRef] [PubMed]

12. Brüggemann, M.; White, H.; Gaulard, P.; Garcia-Sanz, R.; Gameiro, P.; Oeschger, S.; Jasani, B.; Ott, M.; Delsol, G.; Orfao, A.; et al. Powerful strategy for polymerase chain reaction-based clonality assessment in T-cell malignancies Report of the BIOMED-2 Concerted Action BHM4 CT98-3936. Leukemia 2006, 21, 215-221. [CrossRef] [PubMed]

13. Chapuy, B.; Stewart, C.; Dunford, A.J.; Kim, J.; Kamburov, A.; Redd, R.A.; Lawrence, M.S.; Roemer, M.G.M.; Li, A.J.; Ziepert, M.; et al. Molecular subtypes of diffuse large B cell lymphoma are associated with distinct pathogenic mechanisms and outcomes. Nat Med. 2018, 24, 679-690. [CrossRef] [PubMed]

14. Da Silva Almeida, A.C.; Abate, F.; Khiabanian, H.; Martinez-Escala, E.; Guitart, J.; Tensen, C.P.; Vermeer, M.H.; Rabadan, R.; Ferrando, A.; Palomero, T. The mutational landscape of cutaneous T cell lymphoma and Sezary syndrome. Nat Genet. 2015, 47, 1465-1470. [CrossRef] [PubMed]

15. Schmitz, R.; Wright, G.W.; Huang, D.W.; Johnson, C.A.; Phelan, J.D.; Wang, J.Q.; Roulland, S.; Kasbekar, M.; Young, R.M.; Shaffer, A.L.; et al. Genetics and Pathogenesis of Diffuse Large B-Cell Lymphoma. N. Engl. J. Med. 2018, 378, 1396-1407. [CrossRef]

16. Watatani, Y.; Sato, Y.; Miyoshi, H.; Sakamoto, K.; Nishida, K.; Gion, Y.; Nagata, Y.; Shiraishi, Y.; Chiba, K.; Tanaka, H.; et al. Molecular heterogeneity in peripheral T-cell lymphoma, not otherwise specified revealed by comprehensive genetic profiling. Leukemia 2019, 33, 2867-2883. [CrossRef] [PubMed] 